\title{
Simulation of superselective catheterization for cerebrovascular lesions using a virtual injection software
}

Sri Hari Sundararajan ${ }^{{ }^{*}}$ (D), Srirajkumar Ranganathan ${ }^{2}$, Vaishnavi Kishore ${ }^{3}$, Raphael Doustaly ${ }^{3}$ and Athos Patsalides ${ }^{4}$

\begin{abstract}
Background: This report addresses the feasibility of virtual injection software based on contrast-enhanced conebeam CTs (CBCTs) in the context of cerebrovascular lesion embolization. Intracranial arteriovenous malformation (AVM), dural arteriovenous fistula (AVF) and mycotic aneurysm embolization cases with CBCTs performed between 2013 and 2020 were retrospectively reviewed. Cerebrovascular lesions were reviewed by 2 neurointerventionalists using a dedicated virtual injection software (EmboASSIST, GE Healthcare; Chicago, IL). Points of Interest (POIs) surrounding the vascular lesions were first identified. The software then automatically displayed POl-associated vascular traces from vessel roots to selected POls. Vascular segments and reason for POI identification were recorded. Using 2D multiplanar reconstructions from CBCTs, the accuracy of vascular traces was assessed. Clinical utility metrics were recorded on a 3-point Likert scale from 1 (no benefit) to 3 (very beneficial).

Results: Nine cases (7 AVM, 1 AVF, 1 mycotic aneurysm) were reviewed, with 26 POls selected. Three POls were in 2nd order segments, 8 POls in 3rd order segments and 15 POls in 4th order segments of their respective arteries. The reviewers rated all $26 \mathrm{POI}$ traces - involving a total of 90 vascular segments - as accurate. The average utility score across the 8 questions were 2.7 and 2.8 respectively from each reviewer, acknowledging the software's potential benefit in cerebrovascular embolization procedural planning.

Conclusion: The operators considered CBCT-based virtual injection software clinically useful and accurate in guiding and planning cerebrovascular lesion embolization in this retrospective review. Future prospective studies in larger cohorts are warranted for validation of this modality.
\end{abstract}

Keywords: Cerebrovascular lesion, Endovascular embolization, AVM, dAVF, Mycotic aneurysm, Cone-beam CT, CBCT, Embo ASSIST, Vessel detection software, Virtual catheterization, Virtual injection

\section{Background}

Cerebral Arteriovenous Malformations (AVMs), Arteriovenous fistulas (AVFs) and distal aneurysms are dangerous cerebrovascular lesions, potentially leading to intracranial hemorrhage and seizure. They are often managed with endovascular embolization either standalone or in combination with other treatment, such as

\footnotetext{
*Correspondence: sns9009@med.cornell.edu

'Department of Neurosurgery, Division of Interventional Neuroradiology, New York Presbyterian Hospital/Weill Cornell Medical Center, 525 East 68th St, New York, NY 10065, USA

Full list of author information is available at the end of the article
}

microsurgery or stereotactic radiosurgery (Ajiboye et al. 2014; Derdeyn et al. 2017; Jiang et al. 2016). Planning cerebrovascular malformation embolization is challenging because it requires a detailed understanding of both the malformation supplying vasculature anatomy and the arterio-venous flow dynamics (Tranvinh et al. 2017). Cone beam CT (CBCT) is a key modality to overcome the intrinsic limitation of the 2-dimensional (2D) projective nature of DSA and enhances 3D visualization of the anatomy (Doerfler et al. 2015; Honarmand et al. 2015).

\section{Springer Open}

(- The Author(s). 2021 Open Access This article is licensed under a Creative Commons Attribution 4.0 International License, which permits use, sharing, adaptation, distribution and reproduction in any medium or format, as long as you give appropriate credit to the original author(s) and the source, provide a link to the Creative Commons licence, and indicate if changes were made. The images or other third party material in this article are included in the article's Creative Commons licence, unless indicated otherwise in a credit line to the material. If material is not included in the article's Creative Commons licence and your intended use is not permitted by statutory regulation or exceeds the permitted use, you will need to obtain permission directly from the copyright holder. To view a copy of this licence, visit http://creativecommons.org/licenses/by/4.0/. 
Leveraging the $\mathrm{CBCT}$, automatic vessel detection software has been demonstrated to facilitate successful embolization in a wide variety of challenging clinical contexts. Though liver embolization was the primary focus for CBCT-based vessel detection software, it's utility in other anatomic areas have been reported including gastrointestinal arterial bleeds in emergency transarterial embolization (Carrafiello et al. 2016), treatment of renal cell carcinoma, symptomatic benign prostate hypertrophy, small bowel hemorrhage source, and mesenteric pseudoaneurysm (Sundararajan et al. 2019). Safe and effective embolization of challenging lymphatic leakage from the thoracic duct has also been reported when CBCT equipped with guidance software was incorporated (Ierardi et al. 2016). Software integration with addons such as metal artifact reduction can further enhance assessment and awareness when planning embolization procedures (Yuki et al. 2016). Use of such a software has been shown to increase embolization success (Cui et al. 2020), with minimal requirements in additional review and processing time, if the staff is properly trained (Carrafiello et al. 2016).

With past success of vessel detection software reducing fluoroscopy time, contrast use, and improving treatment outcomes, use of this technique for endovascular embolization has potential for smooth transition to treat Cerebrovascular Lesions. This study assesses feasibility and clinical utility of virtual injection software used on CBCTs for intracranial endovascular embolization cases.

\section{Methods}

\section{Patient selection}

Our Institutional Review Board approved this retrospective study. Endovascular embolization of AVMs, dural AVFs, or aneurysms performed at our institution between January 2013 and January 2020 were screened, and procedures with $\mathrm{CBCTs}$ performed during these procedures were included. Patients with lesions in the proximal vasculature such as saccular aneurysms of the Circle of Willis were excluded from the analysis as navigation is typically not challenging. Procedures that had CBCTs with poor image quality caused by motion were also excluded from this review.

\section{Image acquisition}

All patients underwent endovascular mapping or treatment for intracranial embolization in a biplanar angiographic suite (Innova IGS 630, GE Healthcare, Chicago, IL). CBCTs were acquired during cases in which additional 3-dimensional information about the lesion was required. Selective catheterization under fluoroscopic guidance of the right or left internal carotid or vertebral arteries was performed based on the lesion location. Once the desired point of injection was reached, a DSA or a subtracted fluoroscopic loop was acquired to check catheter position. The CBCT was subsequently acquired using a power-injector to maintain constant injection rates of $4 \mathrm{ml} / \mathrm{s}$ during the spin. Three-hundred fortyseven projections were acquired over the $7 \mathrm{~s}$ rotation and were transferred to a workstation for $512 \times 512$ axial reconstructions.

\section{Virtual injection software}

Virtual injection software (Embo ASSIST, GE Healthcare; Chicago, IL) has been developed to simulate selective catheterization from a proximal CBCT (Soliman et al. 2019). After semi-automatic extraction of the vascular tree (Fig. 1a), the user can dynamically simulate any distal catheterization of the vasculature using the mouse pointer as a virtual microcatheter tip. As the pointer is used to select a location in the vasculature, a virtual trace of the vessel proximal to the pointer is instantly created in red (Fig. 1b-c). It represents the virtual catheter navigation from the point of injection to the location selected via the mouse pointer. At the same time, all vessels distal to the pointer are highlighted in green (Fig. 1d-e), mimicking an injection from this point both on the volume rendered vascular tree and on crosssectional views.

\section{Software performance and clinical utility evaluation}

All included patients' studies were transferred to a workstation (Advantage Windows, GE Healthcare, Chicago, IL) for analysis. Two neuro interventionalists, with 15 years (Reviewer A) and 5 years (Reviewer B) respective experience processed independently the $\mathrm{CBCT}$ using the virtual injection software. Semi-automatic extraction of the vascular tree was performed and adjusted by the user if needed. Virtual Injection (VI) feature was then used to simulate different treatment scenarios.

Each neuro-interventionalist identified Points of Interest (POIs) using virtual catheterization on the volume rendered vascular tree. A POI was defined by its segment location on a specific artery. Thereby, POIs selected by the different reviewers on the same segment from the same artery were considered equivalent, even if their 3-dimensional locations were slightly different. Conventional vessel naming was used in denoting POI location with rising order from 1st to 4th order denoting rising levels of vessel distality. For each reviewer, selected POIs were characterized by their clinical purpose for identification and segmental location. POIs whose proximal vessels exited the CBCT field of view were excluded.

The virtual trace associated with each selected POI was analyzed on the CBCT cross-sectional views to assess trace accuracy for each vascular segment, from the most proximal one to the most distal one (Fig. 1e). The 

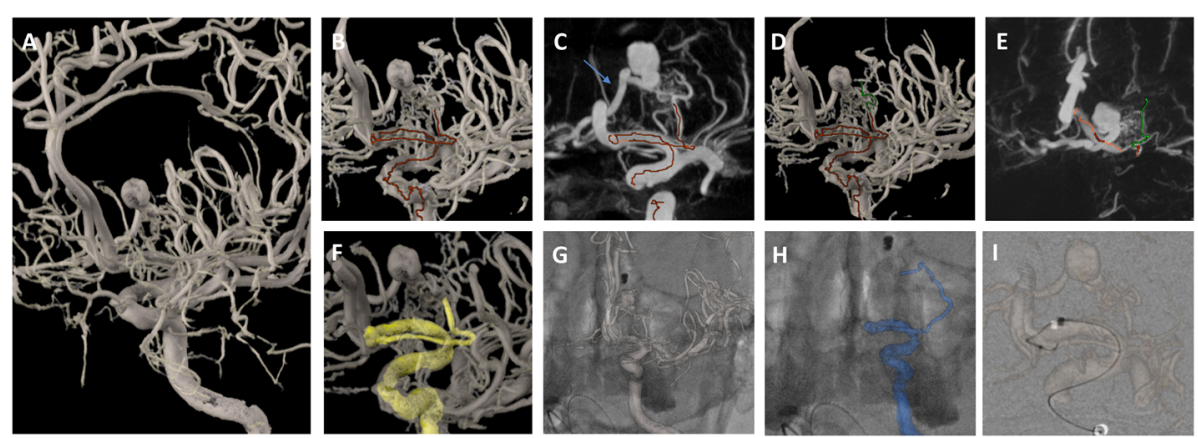

Fig. 1 Use of virtual injection software in neurointerventional radiology. Patient with an AVM the left medial frontal lobe with subjacent flowrelated aneurysm. The vascular tree was automatically extracted, and the bones removed (a). The neuro interventionalist used the virtual microcatheter (the red trace) to review the potential AVM feeders on both the 3D volume rendering (b) and the cross-sectional views (c). On initial glance, apparent primary feeder to the AVM seems to be the anterior cerebral artery (c, blue arrow). However, the software accurately identified this as an en passage vessel supplying the flow-related aneurysm, with subsequent correct identification of the true AVM feeders arising from small caliber lenticulostriate vessels (c-d, red line). Even though it was also possible to simulate injections from the same points (additional distal contrast flow is simulated past the red line in green, $\mathbf{d}$-e), the interventionalist were less interested by this feature as the software prediction are unreliable inside the AVM. The simulated catheterizations were saved as 3D model (f), which could be used in addition to the full vascular in augmented fluoroscopy ( $\mathbf{g}$ - full vascular tree; $\mathbf{h}$ - targeted vessel; $\mathbf{i}$ - example of catheterization guidance)

accuracy of each trace was ranked per segment following a 4-points scale described in Table 1.

The trace was then exported as a separate 3D model of the vessel that stretched from the vascular tree root to the chosen POI. This 3D model was highlighted on the vascular tree in the $3 \mathrm{D}$ volume rendering and could be used as augmented fluoroscopy to guide the procedure during a live case (Fig. 1f-i).

A series of clinical utility questions were asked after the review of each case to gauge the potential benefits of virtual catheterization feature on a 3-points Likert scale from 1 - no benefit to 3 - very beneficial. Utility questions are displayed in Table 2.

\section{Statistical analysis}

Analyses were conducted using Microsoft ${ }^{\circ}$ Excel $^{\circ}$ (Build 11,929.20708), and descriptive statistics were used to represent the data. Inter-reader agreement percentage on clinical utility was measured per question and describes the percentage of scores on which both reviewers agreed.

\section{Results}

\section{Patients}

Nine patients with cerebrovascular lesions involving distal vasculature had $\mathrm{CBCTs}$ performed during the endovascular mapping or treatment for intracranial embolization. CBCTs were performed primarily when the operator felt the utility of added cross-sectional vessel imaging would be of benefit in understanding the pathologic vascular anatomy in question. A total of 7 AVM, 1 AVF, and 1 mycotic aneurysm procedures were processed using the virtual injection software (Table 3). No CBCT was excluded due to poor image quality.

\section{Virtual injection software performance}

Twenty-six independent POIs were selected for review by the neurointerventionalists: 3 POIs of 2nd order, 8 POIs of 3rd order and 15 POIs of 4th order in their respective arteries. Eight of the 26 POIs were selected by both reviewers across 6 cases for the purpose of embolization or elimination of a suspect feeder. Reviewer A identified 5 other independent POIs for the purpose of embolization. Reviewer B identified 13 other POIs either for embolization (Sundararajan et al. 2019), to confirm an en passage vessel (Ajiboye et al. 2014), or to eliminate a suspect AVM feeder vessel (Tranvinh et al. 2017). Twenty-six traces to POIs, involving 90 vascular segments were analyzed by the reviewers, as shown in the examples in Figs. 1, 2, and 3. All of them were rated as accurate, meaning that the vessel tracking was

Table 1 Accuracy Classifications for Traced Vessels

\begin{tabular}{ll}
\hline Classification & Definition \\
\hline Accurate & Vessel tracking is correct from the vascular tree root to the POI \\
Clinically Usable & Vessel tracking is correct for major branch points but incorrectly traces distal vessels \\
Not Clinically Usable & Vessel tracking is wrong for major branch points and distal vessels \\
Not Detected & Vessel cannot be tracked using VI \\
\hline
\end{tabular}


Table 2 Clinical Utility Questions

How helpful was VI in distinguishing between close arterial branches?

How helpful would you expect VI to be in decreasing necessary catheterizations?

How helpful would you expect the VI 3D overlay to be in navigating to the POI?

How helpful would you expect VI to be in decreasing contrast usage?

How helpful would you expect VI to be in decreasing the necessity of $2 \mathrm{D}$ roadmapping during procedures?

How helpful would you expect VI to be in improving your ability to select the correct vessels for embolization?

How helpful would you expect VI to be planning a procedure?

Were you able to select (an) embolization point(s) for this case using VI?

estimated correct from the vascular tree root to the POI for reviewers.

\section{Clinical utility}

The average utility score across the 8 questions was 2.7 out of 3 for Reviewer A and 2.8 for Reviewer B, showing that both reviewers retrospectively evaluated Virtual Injection as clinically useful for cases of intracranial embolization.

The Virtual Injection tool was rated most useful for procedure planning and guidance (Table 4). The use of Virtual Injection in distinguishing between close arterial branches, in navigation to the POI, planning procedures and identifying embolization points was rated as very beneficial, with an average score above 2.8 (Inter-reader agreement of $78 \%, 55 \%$ and $66 \%$ respectively). This technology was also rated as valuable to select an embolization point, to decrease the amount of contrast used, the necessity of $2 \mathrm{D}$ roadmapping and the number of catheterizations, with an average score from 2.6 to 2.7 (Inter-reader agreement of $66 \%$ for the 3 questions). The largest difference between the two reviewers' ratings was regarding the utility of the software in navigation to the POI as a 3D overlay. Reviewer A's average rating was a 2.6 and Reviewer B's average rating was a 3. Table 4 shows the average clinical utility scores and inter-reader agreement for the cases.

\section{Discussion}

The development of new devices and embolic agents has expanded the endovascular approach for cerebrovascular malformation treatment (Pierot et al. 2013), whether in preparation for microsurgery (Natarajan et al. 2008), radiosurgery (Blackburn et al. 2011), or occasionally standalone. The interventionalist must know several characteristics of the malformation in order for the highest chance of successful embolization: the exact vascular supply of the malformation, the number of feeders and feeding pedicles, the flow dynamics of the feeders, and
Table 3 Summary Characteristics

\begin{tabular}{|c|c|c|c|}
\hline \multicolumn{4}{|l|}{ Variable } \\
\hline CBCTs (n) & & \multicolumn{2}{|c|}{9} \\
\hline \multirow[t]{5}{*}{ Injection Point } & $\begin{array}{l}\text { Right Internal Carotid } \\
\text { Artery }\end{array}$ & \multicolumn{2}{|c|}{4} \\
\hline & $\begin{array}{l}\text { Left Internal Carotid } \\
\text { Artery }\end{array}$ & \multicolumn{2}{|c|}{2} \\
\hline & Right Vertebral Artery & \multicolumn{2}{|c|}{1} \\
\hline & Left Vertebral Artery & \multicolumn{2}{|c|}{2} \\
\hline & & $\begin{array}{l}\text { Reviewer } \\
\text { A }\end{array}$ & $\begin{array}{l}\text { Reviewer } \\
\text { B }\end{array}$ \\
\hline \multicolumn{2}{|l|}{ POIs (n) } & 13 & 21 \\
\hline \multicolumn{2}{|l|}{$\begin{array}{l}\text { Avg Segmental } \\
\text { Location }\end{array}$} & 3.3 & 3.6 \\
\hline \multicolumn{2}{|l|}{ Total Segments } & 43 & 76 \\
\hline \multirow{4}{*}{$\begin{array}{l}\text { Segmental } \\
\text { Location }\end{array}$} & 1st order & - & - \\
\hline & 2nd order & 2 & 1 \\
\hline & $3 r d$ order & 5 & 6 \\
\hline & 4th order & 6 & 14 \\
\hline \multirow[t]{10}{*}{ Arterial Location } & Posterior Cerebral Artery & 5 & 5 \\
\hline & Middle Cerebral Artery & 2 & 5 \\
\hline & Anterior Cerebral Artery & 2 & 6 \\
\hline & $\begin{array}{l}\text { Superior Cerebellar } \\
\text { Artery }\end{array}$ & - & 2 \\
\hline & $\begin{array}{l}\text { Anterior Choroidal } \\
\text { Artery }\end{array}$ & 1 & 1 \\
\hline & $\begin{array}{l}\text { Posterior Inferior } \\
\text { Cerebellar Artery }\end{array}$ & - & 1 \\
\hline & Inferolateral Trunk & - & 1 \\
\hline & Lenticulostriate Artery & 1 & - \\
\hline & $\begin{array}{l}\text { Posterior } \\
\text { Communicating Artery }\end{array}$ & 1 & - \\
\hline & $\begin{array}{l}\text { Meningohypophyseal } \\
\text { Trunk }\end{array}$ & 1 & - \\
\hline \multirow{3}{*}{$\begin{array}{l}\text { Purpose of } \\
\text { Selection }\end{array}$} & Embolization & 13 & 16 \\
\hline & $\begin{array}{l}\text { Eliminate Suspect } \\
\text { Feeder }\end{array}$ & - & 4 \\
\hline & Confirm En Passage & - & 1 \\
\hline
\end{tabular}

the characteristics of draining vessels or associated aneurysms. Obtaining a clear and exact picture of these characteristics is difficult with especially dense, complex, and very distal lesions, rendering planning and subsequent embolization much more technically challenging.

Any number of feeder arteries may supply a given lesion, comprising two main patterns of flow: the supplying artery either projects a variable number of feeder arteries to the lesion (while the main artery supplies brain tissue past the branch point) or directly supplies the lesion. The former vascular pattern, known as vessel in passage feeders, levies a higher risk of neurological 

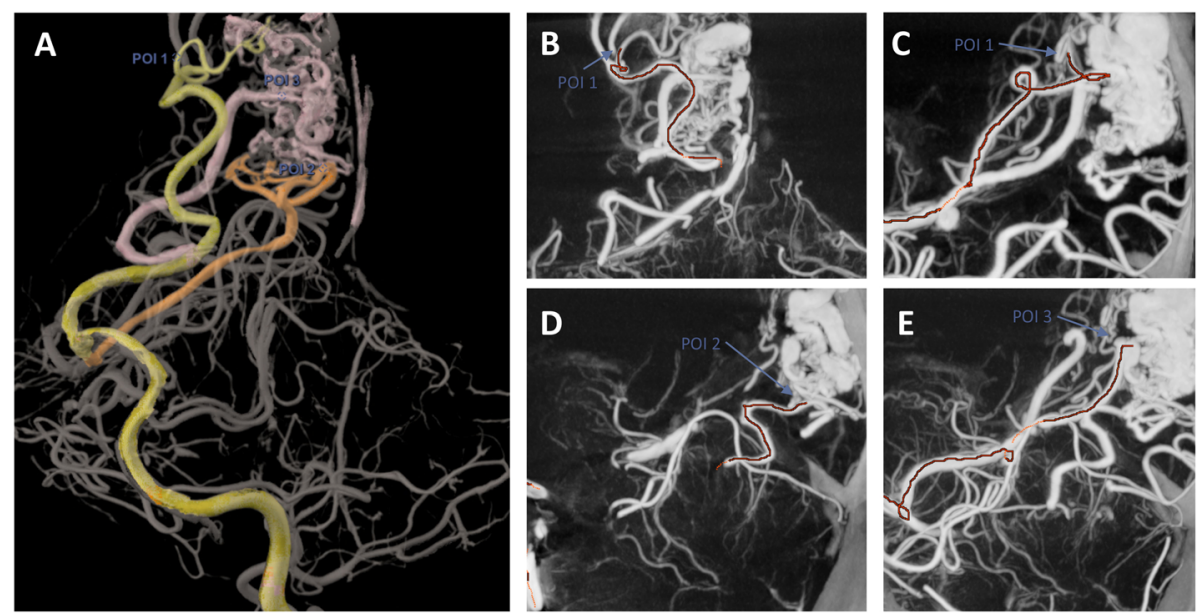

Fig. 2 Retrospective review of an AVM. Each potential AVM supplying vessel was interrogated using virtual catheterization software on the 3D volume rendering. Three Points Of Interest (POls) were identified and exported as 3D models (a). The virtual catherization trace was then reviewed for each POI on cross sectional views to assess its accuracy, as shown in coronal and sagittal reconstruction for POI 1 (b-c), and sagittal reconstructions for $\mathrm{POI} 2$ (b) and $\mathrm{POI} 3$ (c)

deficits after treatment as a result of the risk of embolization or trauma to the main vessel. It is thus is very helpful to be able to visualize single branches of malformations with multiple feeders, elucidating how exactly the malformation receives flow from feeders. When embolizing malformations, it is important to first embolize pedicles that are the largest and/or feed distal veins and aneurysms. Characteristics of the lesion itself also play a crucial role in dictating embolization approach. For instance, more superficial malformations derive their supply most commonly from peripheral branches of the anterior, middle, and/or posterior cerebral arteries, while larger and deeper malformations may derive their supply from lenticulostriates, choroidals, and similarly located central arterial vessels. The deeper the lesion, the more complex and technically difficult the embolization can potentially be due to overlapping of various vasculature on angiographic imaging alone (Crimmins et al. 2015).

Given the need to exactly ascertain these characteristics prior to embolization, the use of advanced imaging technique and $\mathrm{CBCT}$ during embolization of
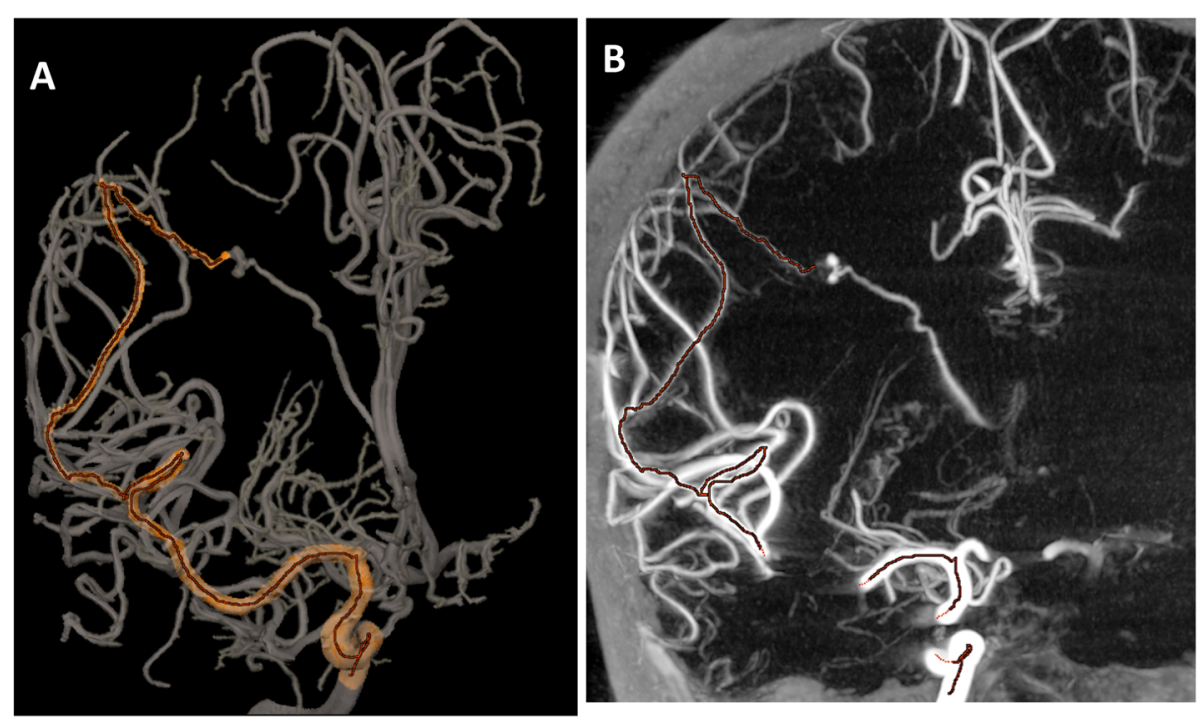

Fig. 3 Retrospective review of a distal aneurysm. Virtual catheterization software was also evaluated for distal embolization, such as in a context of mycotic aneurysm. The software was used to create a 3D roadmap based on the CBCT (a). The accuracy of the 3D roadmap was assessed using the virtual catheterization trace on the cross-sectional views (b) 
Table 4 Clinical Utility Questions and Scores

\begin{tabular}{lll}
\hline Question & $\begin{array}{l}\text { Average Utility Score (Reviewer A Avg, Reviewer B } \\
\text { Avg) }\end{array}$ & $\begin{array}{l}\text { Inter-Reader Agreement } \\
\text { Percentage }\end{array}$ \\
\hline Distinguishing between close arterial branches & $2.9(2.8,3)$ & $77.8 \%$ \\
Decreasing necessary catheterizations & $2.6(2.6,2.6)$ & $66.7 \%$ \\
Navigation to POI as a 3D overlay & $2.8(2.6,3)$ & $55.6 \%$ \\
Decreasing contrast usage & $2.6(2.7,2.6)$ & $66.7 \%$ \\
Decreasing 2D roadmapping during procedures & $2.6(2.7,2.6)$ & $66.7 \%$ \\
Improving ability to select vessels for & $2.7(2.7,2.8)$ & $66.7 \%$ \\
embolization & & \\
Planning a procedure & $2.8(2.8,2.8)$ & $66.7 \%$ \\
Identification of embolization point(s) & $2.8(2.8,2.8)$ & $66.7 \%$ \\
\hline
\end{tabular}

cerebrovascular lesions has exploded in recent years. Lin et al. presented time-resolved $\mathrm{CBCT}$ as a technology providing both anatomic and hemodynamic evaluations for real-time classification of AVMs (Lin et al. 2018), and Sandovia-Garcia et al. suggested that this technique could replace conventional CBCT and multiple 2D DSA in diagnostic and therapeutic procedures (Sandoval-Garcia et al. 2017). In their study, Blanc et al. have demonstrated the applicability of CBCT-based 3D-Roadmap for superselective catheterization during AVM embolization (Blanc et al. 2015).

To the best of our knowledge, this retrospective study is the first one to assess a newly released virtual injection software in a context of cerebrovascular lesion diagnosis or treatment. Compared to the other advanced visualization techniques listed above, virtual injection software is designed to minimize CBCT review time and microcatheter manipulations, by simulating selective catheterizations. All superselective catheterizations simulations in this study were evaluated as accurate, even for the more distal segments. This preliminary result suggests that the application of the virtual catherization technology is safe in cerebral CBCTs.

There were minor differences between Reviewer A (15 years of experience) and Reviewer B (5 years of experience). Selective catheterizations simulations seem more useful for less experienced operators, as illustrated by the increased number of POIs selected by Reviewer B and his higher average utility score compared to Reviewer A. Overall however, both neurointerventionalists described virtual catheterization technology as very useful. Potential benefits were identified in procedure planning: identification of the supplying branches, embolization points and minimizing microcatheter manipulations. These observations corroborate the results published for other anatomies, such as trans-arterial liver-directed therapies, where similar technologies have been available for years (Cui et al. 2020). The software was also evaluated as useful for procedure guidance and to segment the targeted vessels in preparation of an overlay to be used with live fluoroscopy. A few studies reported the benefit of augmented fluoroscopy for neurointerventional procedures in increasing operator confidence, reducing radiation dose, and reducing contrast injected into the patient. In these publications, volumes used for 3D-roadmapping were mainly extracted from pre-op imaging (Zhang et al. 2017; Ruijters et al. 2011; Zhang et al. 2016; Kishore et al. 2020), with no time constraint to segment the structures of interest, but inherent risks of misregistration due to change in patient position. In some other studies, the whole vasculature from the CBCT was used as overlay (Blanc et al. 2015; Jang et al. 2016), as careful segmentation of the targeted vessels was too time-consuming to be performed intraoperatively. In this scenario, the interest in using augmented fluoroscopy might be limited due to many vessels overlapping on the $3 \mathrm{D}$ roadmap and causing cluttered visualization.

The software has certain limitations. The software is FDA approved for utilization only with angiography and cone-beam CT data acquired on GE hardware. However, the software can be utilized on any single plane and biplane GE angiography suite regardless of its age or generation as long as it is a device with cone-beam CT acquisition capability. An artificially wrong course can potentially be mapped if there is significant venous contamination during the cone-beam $\mathrm{CT}$ as subjacent opacified venous structures would interfere with the software's ability to detect clear arterial trajectories. This can also been seen if there is broad or excessive arteriovenous shunting proximal to the malformation's nidus. As such, the software's utilization is best exemplified in clinical situations in which navigation towards a distal aneurysm or focal AVM nidus (and potentially an arteriovenous fistula communication point) without excessive surrounding venous contamination proximal to the target is needed.

This study had notable limitations. The retrospective design did not allow us to assess the software performance in real time procedure settings. The small number 
of patients included is another concern. The small field of view is an intrinsic limitation of such CBCT based vessel detection software, as the software does not track all vessels exiting the CBCT volume. Another common reason given by the reviewers for low utility scores is some patients' straightforward anatomy, where a quick review of $\mathrm{CBCT}$ alone was enough to complete the procedure effectively and efficiently. Additionally, clinical judgment is warranted to select the utility of performing $\mathrm{CBCT}$ in a given cerebrovascular malformation, as each patient's respective anomaly may possess varying degrees of vessel friability and highly suspicious features (such as flow-rated or intra-nidal aneurysms) making them prone to vessel injury ranging from spasm to potential rupture. Such risks may be mitigated with alterations in acquisition technique. These include allowing a rise to achieving final rate of contrast injection, injecting from a common carotid artery rather than direct internal carotid, thereby distributing the total volume of contrast across other surrounding vascular territories (though this may degrade the ability of the software to identify target vessels in the setting of more surrounding opacified vessels).

We believe virtual injection software will promote the adoption of augmented fluoroscopy by enabling rapid segmentation of the targeted vessels. Specifically, this technology adds value via its capability of identifying the appropriate vascular course on 3D images with subsequent allowance for use of the selected route to navigate more efficiently during real-time catheterization and angiography. This software had the potential to be useful not only in the navigation to distal aneurysms and AVM niduses. Precise target vessel selection during catheter directed delivery of chemotherapeutic or immunomodulating agents to intracranial neoplasia is one possible utility of this software, noting that the added reassurance of accurate vessel supply to a given lesion has been of great clinical benefit in the interventional oncology realm of non-neural axis viscera (Cui et al. 2020). Similarly, navigation to branches of the external carotid artery during embolization of head and neck region neoplasia, vascular malformations, or arteriovenous fistula feeders is also technically feasible, noting software limitations that may arise as described earlier.

\section{Conclusion}

In conclusion, virtual catheterization technology simulates accurately selective catheterization in cerebral CBCT. It was considered clinically useful in guiding and planning cerebrovascular lesion embolization by the operators in this retrospective review. As such, this software can potentially be useful for operators with less experience in navigating to distal targets with several possible vascular trajectories on conventional angiography. Future prospective studies in larger cohort are warranted for validation of this modality.

\section{Abbreviations \\ 2D: 2 Dimensional; 3D: 3 Dimensional; AVM: Arteriovenous Malformation; AVF: Arteriovenous Fistula; CBCT: Cone Beam Computed Tomography; POI: Point of Interest}

\section{Acknowledgements}

Not applicable.

\section{Authors' contributions}

SHS analyzed and interpreted data from the study and was a major contributor in writing the manuscript. SR analyzed and interpreted data from the study and was a major contributor in writing the manuscript. VK analyzed and interpreted data from the study and was a major contributor in writing the manuscript. RD analyzed and interpreted data from the study and was a major contributor in writing the manuscript. AP analyzed and interpreted data from the study and was a major contributor in writing the manuscript. All authors read and approved the final manuscript.

\section{Funding}

There is no funding to report for this manuscript.

\section{Availability of data and materials}

The datasets used and/or analyzed during the current study are available from the corresponding author on reasonable request.

\section{Declarations}

\section{Ethics approval and consent to particitapate}

This single center retrospective study was approved by our IRB, and informed written consent was obtained at the time of initial procedural consent.

\section{Consent for publication \\ Consent for publication was obtained at the time of initial procedural content.}

\section{Competing interests}

Our institution has a master research agreement with GE Healthcare. VK and $\mathrm{RD}$ are GE Healthcare employees. Other authors have no conflicts of interest to declare.

\section{Author details}

'Department of Neurosurgery, Division of Interventional Neuroradiology, New York Presbyterian Hospital/Weill Cornell Medical Center, 525 East 68th St, New York, NY 10065, USA. ${ }^{2}$ Northwestern University Feinberg School of Medicine, 420 East Superior Street, Chicago, IL 60611, USA. ${ }^{3}$ GE Healthcare, 500 West Monroe Street, Chicago, IL 60661, USA. ${ }^{4}$ Department of Neuro-Interventional Surgery, North Shore University Hospital, Northwell Health, 300 Community Drive, Manhasset, NY 11030, USA.

Received: 26 March 2021 Accepted: 1 June 2021

Published online: 14 June 2021

\section{References}

Ajiboye N, Chalouhi N, Starke RM, Zanaty M, Bell R (2014) Cerebral arteriovenous malformations: evaluation and management. Sci World J 2014:649036

Blackburn SL, Ashley WW Jr, Rich KM et al (2011) Combined endovascular embolization and stereotactic radiosurgery in the treatment of large arteriovenous malformations. J Neurosurg 114(6):1758-1767. https:/doi.org/1 0.3171/2011.1.JNS10571

Blanc R, Seiler A, Robert T, Baharvahdat H, Lafarge M, Savatovsky J, Hodel J, Ciccio G, Chauvet D, Pistocchi S, Bartolini B, Redjem H, Piotin M (2015) Multimodal angiographic assessment of cerebral arteriovenous malformations: a pilot study. J Neurointerv Surg 7(11):841-847. https://doi.org/10.1136/ neurintsurg-2014-011402

Carrafiello G, lerardi AM, Duka E, Radaelli A, Floridi C, Bacuzzi A, de Bucourt M, de Marchi G (2016) Usefulness of cone-beam computed tomography and automatic vessel detection software in emergency transarterial embolization. 
Cardiovasc Intervent Radiol 39(4):530-537. https://doi.org/10.1007/s00270-01 5-1213-1

Crimmins M, Gobin YP, Patsalides A, Knopman J (2015) Therapeutic management of cerebral arteriovenous malformations: a review. Expert Rev Neurother 15(12):1433-1444. https://doi.org/10.1586/14737175.2015.1079129

Cui Z, Shukla PA, Habibollahi P, Park HS, Fischman A, Kolber MK. A systematic review of automated feeder detection software for locoregional treatment of hepatic tumors. Diagn Interv Imaging. 2020;101(7-8):439-449. https://doi. org/10.1016/j.diii.2020.01.011. Epub 2020 Feb 5

Derdeyn CP, Zipfel GJ, Albuquerque FC, Cooke DL, Feldmann E, Sheehan JP, Torner JC, American Heart Association Stroke Council (2017) Management of brain arteriovenous malformations: a scientific statement for healthcare professionals from the American Heart Association/American Stroke Association. Stroke. 48(8):E200-E224. https://doi.org/10.1161/STR. 0000000000000134

Doerfler A, Gölitz P, Engelhorn T, Kloska S, Struffert T (2015) Flat-panel computed tomography (DYNA-CT) in neuroradiology. from high-resolution imaging of implants to one-stop-shopping for acute stroke. Clin Neuroradiol 25(Suppl 2): 291-297

Honarmand AR, Gemmete JJ, Hurley MC, Shaibani A, Chaudhary N, Pandey AS, Bendok BR, Ansari SA (2015) Adjunctive value of intra-arterial cone beam CT angiography relative to DSA in the evaluation of cranial and spinal arteriovenous fistulas. J Neurointerv Surg 7(7):517-523. https://doi.org/10.113 6/neurintsurg-2014-011139

lerardi AM, Pappalardo V, Liu X, Wu CW, Anuwong A, Kim HY, Liu R, Lavazza M, Inversini D, Coppola A, Floridi C, Boni L, Carrafiello G, Dionigi G (2016) Usefulness of CBCT and guidance software for percutaneous embolization of a lymphatic leakage after thyroidectomy for cancer. Gland Surg 5(6):633-638. https://doi.org/10.21037/gs.2016.12.13

Jang DK, Stidd DA, Schafer S, Chen M, Moftakhar R, Lopes DK (2016) Monoplane $3 \mathrm{D}$ overlay roadmap versus conventional biplane $2 \mathrm{D}$ roadmap technique for neurointervenional procedures. Neurointervention. 11(2):105-113. https://doi. org/10.5469/neuroint.2016.11.2.105

Jiang B, Paff M, Colby GP, Coon AL, Lin LM (2016) Cerebral aneurysm treatment: modern neurovascular techniques. Stroke Vasc Neurol 1(3):93-100. https:// doi.org/10.1136/svn-2016-000027

Kishore V, Sundararajan SH, Doustaly R, Michael M, Xuan D, Link T, Rapoport B, Patsalides A (2020) Magnetic resonance venography for 3-dimensional live guidance during venous sinus stenting. CVIR Endovasc 3(1):66. https://doi. org/10.1186/s42155-020-00158-7

Lin CJ, Yang HC, Chien AC, Guo WY, Wu CC, Hung SC, Chen KK, Wu HM, Luo CB, Chu WF, Hong JS, Wu CSF (2018) In-room assessment of intravascular velocity from time-resolved rotational angiography in patients with arteriovenous malformation: a pilot study. J Neurointerv Surg 10(6):580-586. https://doi.org/10.1136/neurintsurg-2017-013387

Natarajan SK, Ghodke B, Britz GW, Born DE, Sekhar LN (2008) Multimodality treatment of brain arteriovenous malformations with microsurgery after embolization with onyx: single-center experience and technical nuances. Neurosurgery. 62(6):1213-1225; Discussion 1225-1216. https://doi.org/10.122 7/01.neu.0000333293.74986.e5

Pierot L, Cognard C, Herbreteau D, Fransen H, van Rooij WJ, Boccardi E, Beltramello A, Sourour N, Kupcs K, Biondi A, Bonafé A, Reith W, Casasco A (2013) Endovascular treatment of brain arteriovenous malformations using a liquid embolic agent: results of a prospective, multicentre study (BRAVO). Eur Radiol 23(10):2838-2845. https://doi.org/10.1007/s00330-013-2870-6

Ruijters D, Homan R, Mielekamp P, Van De Haar P, Babic D (2011) Validation of $3 \mathrm{D}$ multimodality roadmapping in interventional neuroradiology. Phys Med Biol 56(16):5335-5354. https://doi.org/10.1088/0031-9155/56/16/017

Sandoval-Garcia C, Yang P, Schubert T, Schafer S, Hetzel S, Ahmed A, Strother C (2017) Comparison of the diagnostic utility of 4D-DSA with conventional 2Dand 3D-DSA in the diagnosis of cerebrovascular abnormalities. AJNR Am J Neuroradiol 38(4):729-734. https://doi.org/10.3174/ajnr.A5137

Soliman MM, Stein SI, Doustaly R, Madoff DC (2019) Use of virtual injection software to aid in microcatheter positioning during transarterial chemoembolization. J Vasc Interv Radiol 30(10):1646-1648. https://doi.org/1 0.1016/j.jvir.2019.07.003

Sundararajan SH, Mcclure TD, Winokur RS, Kishore SA, Madoff DC (2019) Extrahepatic clinical application of vessel tracking software and 3D roadmapping tools: preliminary experience. J Vasc Interv Radiol 30(7):10211026. https://doi.org/10.1016/j.jvir.2018.11.039
Tranvinh E, Heit JJ, Hacein-Bey L, Provenzale J, Wintermark M (2017) Contemporary imaging of cerebral arteriovenous malformations. AJR Am J Roentgenol 208(6):1320-1330. https://doi.org/10.2214/AJR.16.17306

Yuki I, Kambayashi Y, Ikemura A, Abe Y, Kan I, Mohamed A, Dahmani C, Suzuki T, Ishibashi T, Takao H, Urashima M, Murayama Y (2016) High-resolution C-arm $C T$ and metal artifact reduction software: a novel imaging modality for analyzing aneurysms treated with stent-assisted coil embolization. AJNR Am J Neuroradiol 37(2):317-323. https://doi.org/10.3174/ajnr.A4509

Zhang Q, Sun Q, Zhang Y, Zhang H, Shan T, Han J, Pan W, Gu C, Xu R (2017) Three-dimensional image fusion of CTA and angiography for real-time guidance during neurointerventional procedures. J Neurointerv Surg 9(3): 302-306. https://doi.org/10.1136/neurintsurg-2015-012216

Zhang Q, Zhang Z, Yang J, Sun Q, Luo Y, Shan T, Zhang H, Han J, Liang C, Pan W, Gu C, Mao G, Xu R (2016) CBCT-based 3D MRA and angiographic image fusion and MRA image navigation for neuro interventions. Medicine (Baltimore) 95(32):E4358. https://doi.org/10.1097/MD.0000000000004358

\section{Publisher's Note}

Springer Nature remains neutral with regard to jurisdictional claims in published maps and institutional affiliations.

\section{Submit your manuscript to a SpringerOpen ${ }^{\circ}$ journal and benefit from:}

- Convenient online submission

- Rigorous peer review

- Open access: articles freely available online

- High visibility within the field

- Retaining the copyright to your article

Submit your next manuscript at $\boldsymbol{\nabla}$ springeropen.com 